\title{
CAD-Based Monte Carlo Neutron Transport Analysis for KSTAR
}

\author{
Geon Ho Seo, Sung Hoon Choi and Hyung Jin Shim* \\ Nuclear Engineering Department, Seoul National University, 08826 Seoul, Korea
}

\begin{abstract}
The Monte Carlo (MC) neutron transport analysis for a complex nuclear system such as fusion facility may require accurate modeling of its complicated geometry. In order to take advantage of modeling capability of the computer aided design (CAD) system for the MC neutronics analysis, the Seoul National University MC code, McCARD, has been augmented with a CAD-based geometry processing module by imbedding the OpenCASCADE CAD kernel. In the developed module, the CAD geometry data are internally converted to the constructive solid geometry model with help of the CAD kernel. An efficient cell-searching algorithm is devised for the void space treatment. The performance of the CAD-based McCARD calculations are tested for the Korea Superconducting Tokamak Advanced Research device by comparing with results of the conventional MC calculations using a text-based geometry input.
\end{abstract}

\section{Introduction}

The Monte Carlo (MC) particle transport analysis for a complex system such as an accelerator or a fusion facility may require accurate modeling of the complicated geometry. However its manual modeling of all the geometrical objects in a text input file is lengthy and error-prone. In order to overcome this problem, techniques for automatic coupling of MC codes with the computer aided design (CAD) system have been widely studied and applied for various transport analyses [1-6].

There have been two kinds of approaches to develop MC code systems utilizing the CAD data: the external format conversion and the CAD kernel imbedded MC simulation. The first approach includes several interfacing programs such as McCad [1], MCAM [2], GEOMIT [3] etc. which were developed to automatically convert the CAD data into the MCNP [7] geometrical input data. This approach makes the most of the existing MC codes without any modifications, but implies latent data inconsistency due to the difference of the geometry modeling system. In the other approach, a MC code directly utilizes the CAD data for the MC particle tracking or an internal conversion to an internal data structure of the constructive solid geometry (CSG) and/or boundary representation (B-rep) modeling with help of an imbedded CAD kernel. MCNP-BRL [4], DAGMC [5], and OiNC [6] have demonstrated their capabilities of the CAD-based MC simulations.

The Seoul National University MC code, McCARD [8], has demonstrated [9] its CAD-based MC neutronics analysis capability for the HANARO research reactor [10]. In McCARD, the CAD geometrical data are automatically converted into the internal geometrical data structure with help of an imbedded CAD kernel, OpenCASCADE (OCC) [11]. Recently authors have augmented a McCARD version with the CAD-based geometry processing module (or McCARD/CAD) by an efficient algorithm for the void space treatment required for the fusion facility analysis. In this paper, we examine the performance of McCARD/CAD for the neutronics analysis of Korea Superconducting Tokamak Advanced Research (KSTAR) device [12].

\section{Geometry Processing in McCARD/CAD}

\subsection{Distance to Surface Calculation Module}

In a course of the MC particle tracking, geometrical modeling data are required to calculate the minimum distance to surface (DTS) from a particle at a given location and direction in a cell. For efficient DTS computations, cells bounded by elementary surfaces such as planar, cylindrical, spherical, conical, and toroidal surfaces in the CAD geometrical data file are converted into the internal geometrical data structure in McCARD. When a solid surface is not the elementary such as a Bspline or a rotational one, then OCC library functions are directly utilized to calculate the DTS. Figure 1 shows an algorithm of the McCARD/CAD DTS calculation.

\footnotetext{
* Corresponding author: shimhj@snu.ac.kr
} 


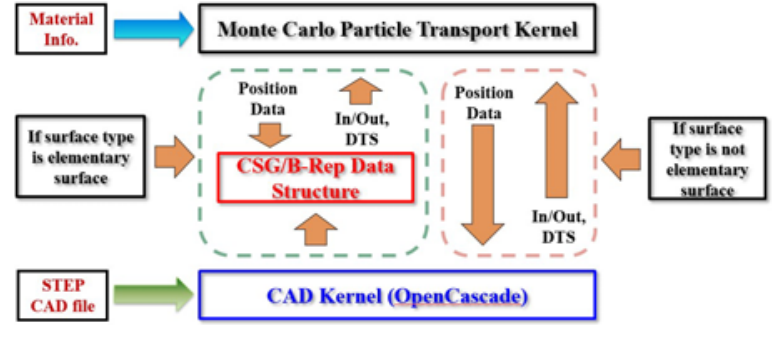

Fig. 1. Algorithm of McCARD/CAD DTS calculation

\subsection{Cell Conversion}

In the McCARD CAD-based geometry processing module, a solid in the CAD model whose boundaries are represented by elementary surfaces such as plane, cylindrical, spherical, conical, toroidal surfaces is converted into the McCARD cell model in which a cell is defined by Boolean operations such as 'AND', 'OR', 'NOT' of the boundary surfaces. For this cell conversion, the following geometrical information for each solid is required.

- surface equations of solid boundaries

- direction $(+,-)$ of each boundary surface including the solid

- Boolean operators ('AND' or 'OR') between surfaces

The surface equations and directions of bounding surfaces can be readily obtained by the OCC library functions. In order to represent a concave-type solid into a union of convex-type cells in which the surfaces are connected by the 'AND' operator, the following algorithms are used.

(1) Find all vertices which make up a solid.

(2) If the vertices are located only in one side of a plane which bounds the solid, then the plane surface is assigned to convex-type. If not, the plain becomes concave-type.

(3) If a plane is concave-type, divide the solid by the plane and connect the divided solids by the union ('OR') operator.

(4) Repeat the process step (1) (3) until all the divided solids becomes convex.

(5) Define the divided convex solid by the intersections of its boundary surfaces using the 'AND' operators and combine all the divided convex solids with the 'OR' operators

\subsection{Void Space Treatments}

An artificial void cell per each subsystem which can access all the surface data of the subsystem is automatically created in McCARD/CAD to simulate particle tracks in its void spaces. When a particle enters the void cell, the minimum distance to surface is selected among distances to all the surfaces with checking their validities. In order to enhance the efficiency, a cell which the particle enters crossing the selected surface is searched by priorities of neighbor cells which are updated during the MC particle tracking.

\section{Application for KSTAR}

The neutron flux of the KSTAR fusion research device [11] is calculated by McCARD/CAD. Figure 2 shows the CAD model of KSTAR used in the McCARD/CAD calculations. The KSTAR CAD model consists of plasma facing components, a vacuum vessel, poloidal field coils, and toroidal field coils. For the comparison, the McCARD calculations using text geometry inputs are also conducted with or without the void space treatments. The McCARD calculations are performed on one billion neutron sources with the Gaussian fusion energy spectrum using the ENDF/B-VII.0 continuous energy cross section libraries.

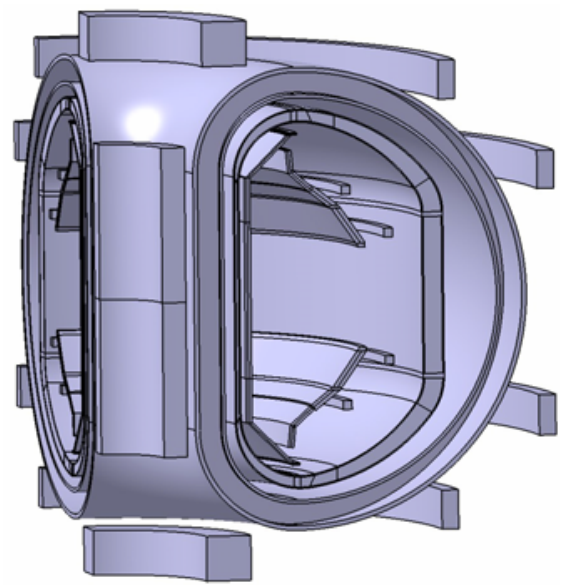

Fig. 2. CAD model of KSTAR

Figures 3 and 4 show the comparisons of neutron fluxes in the vacuum vessel and the toroidal field coil, respectively, calculated by $\mathrm{McCARD} / \mathrm{CAD}$ and McCARD using a text input file. In the figures, the $x$ axis denotes the angular location of the vessel or coil. From the figures, we can see that the flux results are identical within their statistical confidence intervals. Table 1 shows the comparison of elapsed times of three McCARD calculations. From the table, we can observe that the McCARD/CAD with the void space treatment takes about $57 \%$ additional times comparing to the McCARD without the void space treatment. 


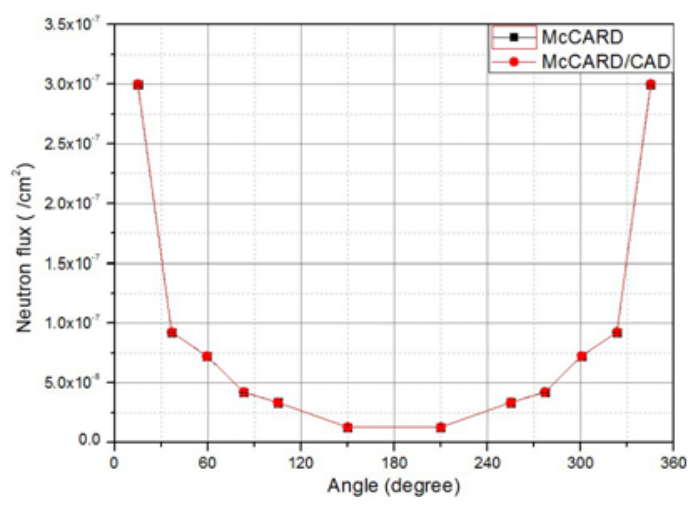

Fig. 3. Comparison of neutron flux in the vacuum vessel calculated by McCARD/CAD and McCARD

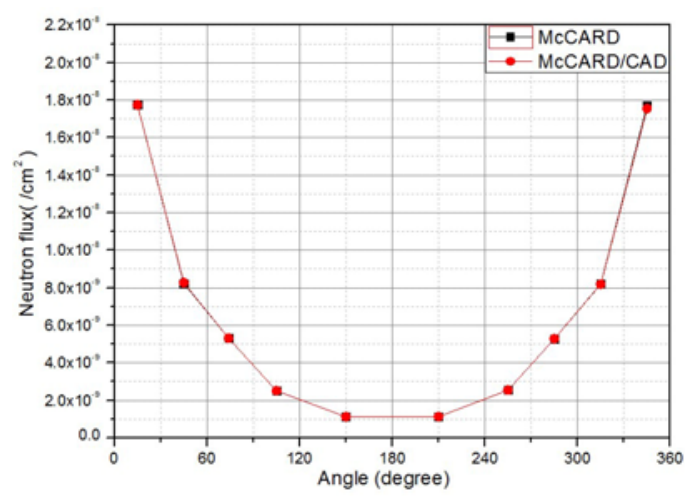

Fig. 4. Comparison of neutron flux in the toroidal field coil calculated by McCARD/CAD and McCARD

Table 1. Comparison of McCARD calculation times

\begin{tabular}{|c|c|c|c|}
\hline & $\begin{array}{c}\text { McCARD } \\
\text { /CAD }\end{array}$ & \multicolumn{2}{|c|}{$\begin{array}{c}\text { McCARD } \\
\text { with a text input }\end{array}$} \\
\hline $\begin{array}{c}\text { Void space } \\
\text { treatment }\end{array}$ & on & on & off \\
\hline $\begin{array}{c}\text { CPU time } \\
\text { [hour }]\end{array}$ & 25.31 & 24.73 & 16.07 \\
\hline
\end{tabular}

\section{References}

1. H. Tsige-Tamirat, U. Fischer, A. Serikov, S. Stickel, Fusion Eng. Des., 83, 1771 (2008)

2. Y. Wu and FDS Team, Fusion Eng. Des., 84, 1987 (2009)

3. S. Sato, et al., Nucl. Technol., 168, 843 (2009)

4. K.B. Bekar, T.M. Evans, Trans. Am. Nucl. Soc., 101, 623 (2009)

5. P.P.H. Wilson, et al., Fusion Eng. Des., 85, 1759 (2010)

6. F. Fleurot, K. Searson, "OiNC, Criticality Benchmark Models in CAD," ICNC2011International Conference on Nuclear Criticality, Edinburgh (2011)

7. J. F. Briesmeister, MCNP-A General Monte Carlo $N$-Particle Transport Code, Version 4C, LA-13709, Los Alamos National Laboratory (2000)

8. H.J. Shim, et al., Nucl. Eng. Technol., 44, 161 (2012)
9. H.J. Shim, et al., Ann. Nucl. Energy, 82, 48 (2015)

10. H. Kim, et al., J. Nucl. Sci. Technol., 33, 527-538 (1996)

11. OpenCASCADE ${ }^{\mathrm{TM}}$, www.opencascade.org

12. G.S. Lee, et al., Nucl. Fusion, 40, 575 (2000). 